\title{
The SMART Registry: Long-Term Results on the Utility of the Penumbra SMART COIL System for Treatment of Intracranial Aneurysms and Other Malformations
}

OPEN ACCESS

Edited by:

Ning Lin,

Cornell University, United States

Reviewed by:

Alicia C. Castonguay,

University of Toledo, United States

Nabeel A. Herial,

Thomas Jefferson University,

United States

*Correspondence: Alejandro M. Spiotta spiotta@musc.edu

Specialty section: This article was submitted to Endovascular and Interventional Neurology,

a section of the journal

Frontiers in Neurology

Received: 03 December 2020 Accepted: 12 March 2021

Published: 13 April 2021

Citation:

Spiotta AM, Park MS, Bellon RJ, Bohnstedt BN, Yoo AJ, Schirmer CM,

DeLeacy RA, Fiorella DJ, Woodward BK, Hawk HE, Nanda A, Zaidat OO, Sunenshine PJ, Liu KC, Kabbani MR, Snyder KV,

Sivapatham T, Dumont TM,

Reeves $A R$, Starke RM and the SMART Registry Investigators (2021)

The SMART Registry: Long-Term Results on the Utility of the Penumbra SMART COIL System for Treatment of

Intracranial Aneurysms and Other Malformations.

Front. Neurol. 12:637551.

doi: 10.3389/fneur.2021.637551

\begin{abstract}
Alejandro M. Spiotta ${ }^{\text {* }}$, Min S. Park ${ }^{2}$, Richard J. Bellon ${ }^{3}$, Bradley N. Bohnstedt ${ }^{4}$, Albert J. Yoo ${ }^{5}$, Clemens M. Schirmer ${ }^{6}$, Reade A. DeLeacy ${ }^{7}$, David J. Fiorella ${ }^{8}$, B. Keith Woodward ${ }^{9}$, Harris E. Hawk ${ }^{10}$, Ashish Nanda ${ }^{11}$, Osama O. Zaidat ${ }^{12}$, Peter J. Sunenshine ${ }^{13}$, Kenneth C. Liu ${ }^{14}$, Mouhammed R. Kabbani ${ }^{15}$, Kenneth V. Snyder ${ }^{16}$, Thinesh Sivapatham ${ }^{17}$, Travis M. Dumont ${ }^{18}$, Alan R. Reeves ${ }^{19}$, Robert M. Starke ${ }^{20}$ and the SMART Registry Investigators

${ }^{1}$ Department of Neurosurgery, Medical University of South Carolina, Charleston, SC, United States, ${ }^{2}$ Department of Neurosurgery, University of Virginia, Charlottesville, VA, United States, ${ }^{3}$ Department of Interventional Neuro Radiology, Swedish Medical Center, Denver, CO, United States, ${ }^{4}$ Oklahoma University Medical Center, Oklahoma City, OK, United States, ${ }^{5}$ Department of Interventional Neuroradiology, Texas Stroke Institute, Dallas, TX, United States, ${ }^{6}$ Geisinger Medical Center, Danville, PA, United States, ${ }^{7}$ Mount Sinai, New York, NY, United States, ${ }^{8}$ Stony Brook University Medical Center, Cerebrovascular Center, New York, NY, United States, ${ }^{9}$ Vista Radiology, Knoxville, TN, United States, ${ }^{10}$ Erlanger Health System, Chattanooga, TN, United States, ${ }^{11}$ SSM Health Medical Group, Fenton, MO, United States, ${ }^{12}$ St Vincent Mercy Health Medical Center, Toledo, OH, United States, ${ }^{13}$ Banner University Medical Center, Phoenix, AZ, United States, ${ }^{14}$ Penn State Milton S. Hershey Medical Center, Hershey, PA, United States, ${ }^{15}$ Gundersen Health System, La Crosse, WI, United States, ${ }^{16}$ Department of Neurosurgery, University of Buffalo, Buffalo, NY, United States, ${ }^{17}$ Christiana Care Health System, Newark, DE, United States, ${ }^{18}$ Department of Surgery, University of Arizona, Tucson, AZ, United States,

${ }^{19}$ Department of Radiology, University of Kansas, Kansas City, KS, United States, ${ }^{20}$ Department of Neurological Surgery, University of Miami Hospital, Miami, FL, United States
\end{abstract}

Introduction: Penumbra SMART $\mathrm{COIL}^{\circledR}$ (SMART) System is a novel generation embolic coil with varying stiffness. The study purpose was to report real-world usage of the SMART System in patients with intracranial aneurysms (ICA) and non-aneurysm vascular lesions.

Materials and Methods: The SMART Registry is a post-market, prospective, multicenter registry requiring $\geq 75 \%$ Penumbra Coils, including SMART, PC400, and/or POD coils. The primary efficacy endpoint was retreatment rate at 1-year and the primary safety endpoint was the procedural device-related serious adverse event rate.

Results: Between June 2016 and August 2018, 995 patients (mean age 59.6 years, $72.1 \%$ female) were enrolled at 68 sites in the U.S. and Canada. Target lesions were intracranial aneurysms in $91.0 \%$ of patients; $63.5 \%$ were wide-neck and $31.8 \%$ were ruptured. Adjunctive devices were used in 55.2\% of patients. Mean packing density was $32.3 \%$. Procedural device-related serious adverse events occurred in $2.6 \%$ of patients. The rate of immediate post-procedure adequate occlusion was $97.1 \%$ in aneurysms and the rate of complete occlusion was $85.2 \%$ in non-aneurysms. At 1-year, the retreatment rate was 6.8\%, Raymond Roy Occlusion Classification (RROC) I or II was $90.0 \%$ for aneurysms, and Modified Rankin Scale (mRS) 0-2 was achieved in $83.1 \%$ of all patients. Predictors of 1-year for RROC III or retreatment (incomplete occlusion) were rupture 
status $(P<0.0001)$, balloon-assisted coiling $(P=0.0354)$, aneurysm size $(P=0.0071)$, and RROC III immediate post-procedure $(P=0.0086)$ in a model that also included bifurcation aneurysm $(P=0.7788)$. Predictors of aneurysm retreatment at 1 -year was rupture status $(P<0.0001)$.

Conclusions: Lesions treated with SMART System coils achieved low long-term retreatment rates.

Clinical Trial Registration: https://www.clinicaltrials.gov/, identifier NCT02729740.

Keywords: embolization coil, intracranial aneurysm, SMART COIL, intracranial fistula, intracranial malformations

\section{INTRODUCTION}

Intracranial aneurysm rupture is a catastrophic clinical outcome and occurs annually in $\sim 30,000$ people in the U.S. (1) Endovascular coiling is widely accepted as treatment for intracranial aneurysms following the results from ISAT (2). Endovascular coiling was shown to be safe and efficacious and resulted in less disability than surgical clipping. However, retreatment rate at 1 -year was higher for coiling $(17.4 \%)$ than for surgical clipping (3.8\%) (3). In the decade since the ISAT results were published, advances in coiling technology, including deliverability, materials, and design, have been developed. Endovascular coiling remains a viable treatment alternative to flow diverters and intrasaccular devices.

The Penumbra SMART COIL ${ }^{\circledR}$ (SMART) System is a microcoil system, which includes SMART COIL ${ }^{\circledR}$, Penumbra Coil $400^{\mathrm{TM}}\left(\mathrm{PC} 400^{\mathrm{TM}}\right)$, and Penumbra Occlusive Device ${ }^{\circledR}$ $\left(\mathrm{POD}^{\circledR}\right)$ indicated for endovascular embolization in the peripheral and neuro vasculature. All devices were available in the U.S market at the start of enrollment in 2016. The SMART System's unique design feature is varying levels of softness that increase toward the proximal tip. This progressive softness enhances deliverability in multiple aneurysm sizes and shapes. The SMART System design may improve deployment by stabilizing the microcatheter positioning, compared to other coils with uniform stiffness (4). The unique design of the SMART System may possibly assist with achieving higher packing density and adequate occlusion in multiple aneurysm types.

Other SMART Coil study results reported adequate aneurysm occlusion between 71.2 and $100 \%$ of treated aneurysms, low rebleeds, and low retreatment rates (5-8). Those studies had either small sample sizes, few study sites, or lacked long-term follow-up. The SMART Registry was a prospective, post-market study, designed to evaluate the SMART System in the treatment of intracranial aneurysms and other neurovascular lesions. We report the results of the SMART Registry procedural and long-term study endpoints in patients with intracranial aneurysm or other, non-aneurysm lesions, and investigate predictors of aneurysm retreatment and inadequate occlusion at 1-year. The SMART Registry provides valuable insights on treatment practices in the U.S. and real-world data on common safety and efficacy outcome measures.

\section{MATERIALS AND METHODS}

\section{Design}

The SMART Registry was a prospective, single-arm, post-market, multicenter registry of the SMART System (Penumbra, Inc.). A maximum of 1,000 patients were planned to be enrolled in up to 100 international sites. Patients were treated in accordance with the cleared indications for the SMART, PC 400, and POD for intracranial aneurysms and other, non-aneurysm vascular lesions. The study received approval from the Institutional Review Board (IRB) and Ethics Committee (EC) from each participating site with oversite for the duration of the registry. The study was designed in accordance with the relevant aspects of clinical research regulations. Written informed consent was provided by patients or their legally authorized representative (LAR). For patients that were treated in emergent cases, they were allowed to be enrolled if they signed the consent within one calendar day after the procedure, or if a LAR signed on their behalf. Penumbra, Inc. sponsored the oversite of this trial.

\section{Eligibility Criteria}

Patients included in the SMART Registry were required to have a signed consent form and undergo embolization of an intracranial aneurysm or other neurovascular abnormalities such as an arteriovenous malformations or an arteriovenous fistula (nonaneurysm lesions) according to the cleared indications. Patients were excluded if SMART, PC400, or POD coils accounted for $<75 \%$ of the total number of coils implanted; if their life expectancy was $<1$ year; if they were already enrolled in the SMART Registry, or if they were participating in another investigation(s) that could confound results.

\section{Endpoints}

The primary efficacy endpoint was retreatment at 1-year $( \pm 6$ months), and the primary safety outcome was procedural device-related serious adverse events (SAEs) within $24 \mathrm{~h}$ of the procedural arterial puncture. The secondary efficacy endpoints were ability to achieve adequate occlusion immediate postprocedure and the number of times re-access with the microwire was required due to catheter kickout. Other clinical outcomes were adequate occlusion, recanalization, and modified Rankin Scale (mRS) $0-2$ at 1-year; all-cause mortality within $24 \mathrm{~h}$ of procedure and at 1-year; and all SAEs that were intraprocedural 
or within $24 \mathrm{~h}$ of procedure, after $24 \mathrm{~h}$ and up to 365 days ( \pm 180 days for visits in window).

\section{Procedures and Data Collection}

Demographics and medical history were recorded at the baseline visit and all patients were assessed in accordance with the institutional standard of care. Endovascular embolization procedures were completed per each investigational site's standard of care. Coil diameter and lengths were selected on the basis of aneurysm dimensions and physician preference. All lesions were treated with $\geq 75 \%$ SMART coils. Patients were administered local, conscious, or general sedation per physician preference. Target lesions were accessed by transfemoral, transradial, or other approaches. All adjunctive techniques and devices were permitted. Cerebral angiograms were obtained immediate post-procedure and at 1-year ( \pm 6 months). The aneurysm occlusion status was determined by the treating physician immediately post-procedure and the results were used to compare to the follow-up assessment. From the time of enrollment through study exit, safety endpoints were monitored, and adverse events were collected and assessed for whether they were serious, device related, or procedure related. Study data was collected by investigational sites using Inform electronic data capture (EDC) system.

\section{Study Definitions}

The RROC was used to determine angiographic aneurysm occlusion status, with Class I as complete occlusion, Class II as residual neck, and Class III as residual aneurysm (9). For non-aneurysm lesions, occlusion was recorded as complete or incomplete as determined by the treating physician's assessment of whether blood flow remained on post-procedure imaging. The Hunt and Hess scale (10) was used at admission to determine severity of ruptured aneurysms; all scores were eligible for enrollment. For device-related and procedure-related SAEs, relationship to the device (definite/probable/possible/unrelated) was determined by the investigator. Events reported as "definite, probable, or possible" were classified as "related." Coil packing density was calculated by using either software calculators or by calculating aneurysm volume assuming an ellipsoid model and coil volume $\left[\mathrm{V}=\pi(p / 2)^{2} \times \mathrm{L}\right]$, where $p$ represents primary coil diameter, and $\mathrm{L}$ represents coil length. Packing density was not calculated for patients with deconstructive treatment, fusiform or dissecting aneurysms, or non-aneurysmal lesions. A wide-necked aneurysm was defined as having a dome-to-neck ratio $<2$ or a neck width of at least $4 \mathrm{~mm}$. The Modified Rankin Scale (mRS) was captured at admission and at 1-year if available per the site standard of care.

\section{Statistical Analysis}

The predetermined sample size $(n=1,000)$ allowed the expected retreatment rate of $8.3 \%$ at 1 year to be estimated with $>$ $\pm 2 \%$ precision. Summary statistics for all patients, patients with aneurysm, and patients with non-aneurysm lesions were developed separately. Descriptive statistics with a 95\% twosided confidence interval were presented for most analyses. Continuous variables were summarized with descriptive statistics
[ $n$, mean, standard deviation, median, and interquartile range (IQR)]. For categorical data, frequency counts and percentage of patients within each category were included. Predictive analyses were performed for 1-year outcomes after aneurysm treatment-incomplete occlusion (RROC III or retreatment) and retreatment. The analyses were run by using binary logistic regression models with stepwise selection (alpha-toenter $\leq 0.20$, alpha-to-leave $>0.05$ ). The following variables were considered for predictive models when their univariate logistic regression $p$-value was $\leq 0.20$ : patient age, sex, history of hypertension history of smoking, cocaine use, and family history of aneurysm or malformation; aneurysm type, size, and location; aneurysm status as ruptured, wide-necked, and neck width at least $4 \mathrm{~mm}$; stent-assisted coiling; balloon-assisted coiling; deconstructive treatment; coil packing density; and RROC immediately post-procedure. SAS 9.4 (SAS Institute) was used for statistical programming.

\section{RESULTS}

\section{All Patient Outcomes Demographics and Baseline Characteristics}

Between June 2016 and August 2018, 995 patients were enrolled at 67 US sites (989 patients) and one Canadian site (6 patients). Figure 1 illustrates the study Flow Diagram. Baseline characteristics and medical history for all patients and by aneurysm and non-aneurysm cohort are presented in Table $\mathbf{1 .}$ The target lesion was an intracranial aneurysm in 905 (91.0\%) patients; the remaining 90 (0.9\%) lesion types were arteriovenous fistula, arteriovenous malformation, and vessel sacrifice or other pseudoaneurysm. For the overall population, mean age of patients was 59.6 years (SD 13.00, range $12-93$ years), $72.1 \%$ of the patients were female, $61.2 \%$ had a history of smoking, $60.6 \%$ had a history of hypertension, and $17.1 \%$ had a family history of aneurysm or malformation.

Lesion characteristics are described in Table 2. Most (75.2\%) lesions were located in the anterior circulation. The lesion was on the left side in $35.6 \%$ patients, on the right side in $35.6 \%$ patients, and on the midline in $28.8 \%$ patients. Lesion locations included 3.7\% (37/995) extradural ICA, 33.0\% (328/995) ICA, 26.7\% (52/995) ACA, 11.8\% (117/995) MCA, 18.9\% (188/995) posterior circulation, $2.4 \%$ (24/995) venous circulation, and 3.5\% (35/995) other.

\section{Procedural Characteristics}

Procedural characteristics are described in Table 3. The mean time from first coil deployment to last coil detached was $24.1 \mathrm{~min}$ (SD 25.73) and the median time was 16.0 (IQR 8.0, 32.0).

\section{Performance Outcomes}

Adequate occlusion at immediate post-procedure was achieved in 97.1\% (965/994) of patients. The first framing coil was completely conformed to the lesion morphology in $83.1 \%$ (766/922) of cases. At 1-year, retreatment occurred in 6.8\% (53/784) of patients and $83.1 \%$ (463/557) had mRS 0-2. Patient outcomes are provided in Table 4. 


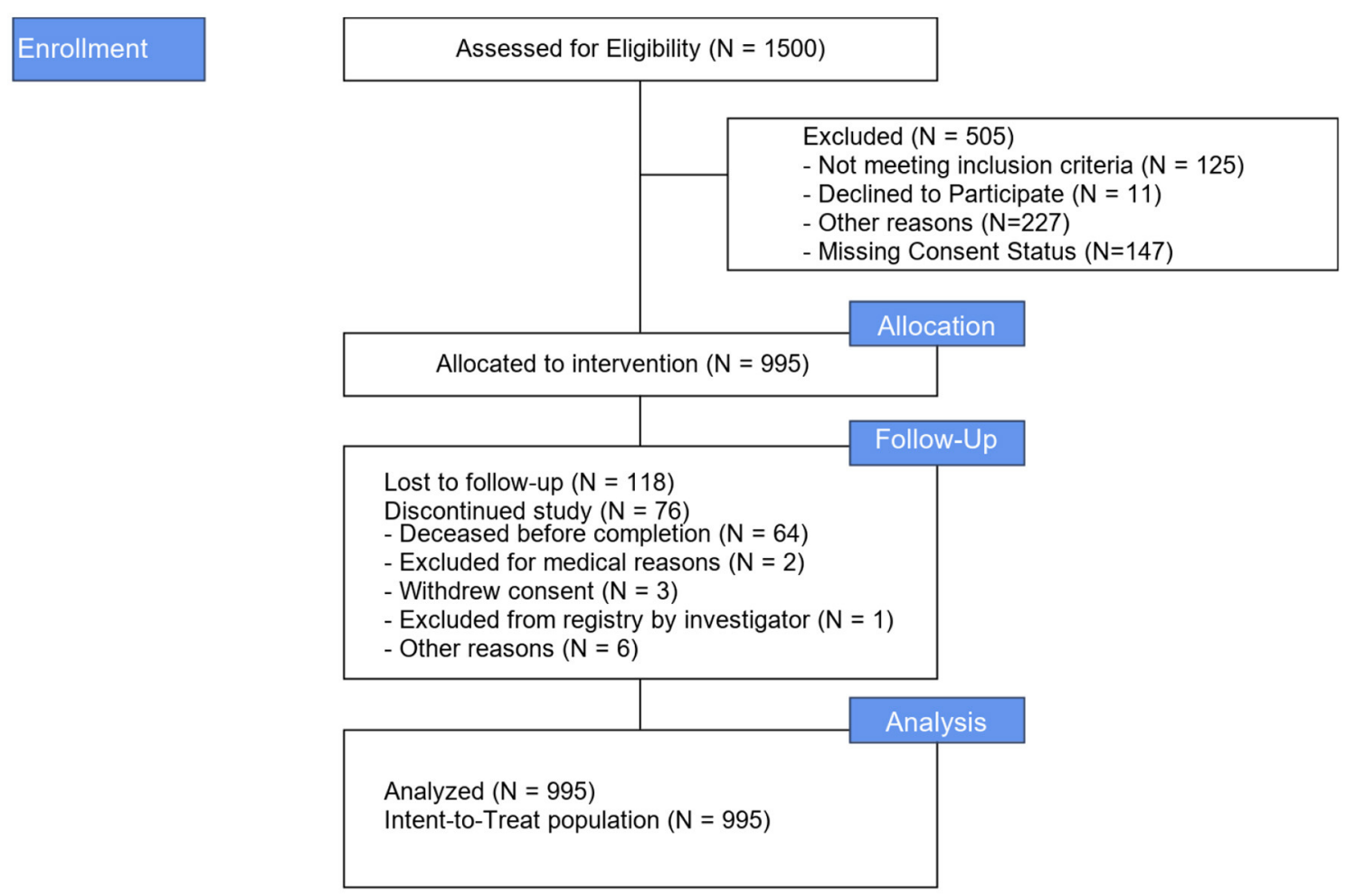

FIGURE 1 | Study flow diagram.

\section{Safety Outcomes}

Procedural device-related SAEs occurred in 2.6\% (26/995) of patients. The rate of device malfunctions associated with an AE was $0.2 \%$ (2/995); one patient experienced an aneurysm perforation and one patient experienced a coil herniation into the parent artery. The overall mortality rate for all patients within $24 \mathrm{~h}$ of the procedure was $0.2 \%(2 / 995)$. Neither of the two deaths were related to the device or the index procedure. The 1year mortality rate was $6.0 \%(60 / 995)$. The SAE rate was $9.4 \%$ (intraprocedural or within $24 \mathrm{~h}$ ) and $22.7 \%$ after $24 \mathrm{~h}$ through 365 days.

\section{Aneurysm Cohort Outcomes \\ Demographics and Baseline Characteristics}

Of the 905 patients treated for aneurysms, the mean age was 59.8 year (SD 12.64), 74.7\% were female, and $78.6 \%$ were white. There were $18.3 \%(166 / 905)$ of patients that had a familial history of aneurysms and $61.8 \%(559 / 905)$ had hypertension.

\section{Procedural Characteristics}

Average aneurysm size was $6.9 \mathrm{~mm}$ (SD 3.62) and $19.0 \%$ of aneurysms were small $(\leq 4 \mathrm{~mm})$. Patient aneurysm types were $86.3 \%$ saccular, $63.5 \%$ wide-necked, and $31.8 \%$ ruptured; $43.5 \%$ of patients with a ruptured aneurysm had Hunt and Hess $\geq 3$ severity. Unassisted coiling (UAC) was used in 43.3\% (392/905) of cases, stent-assisted coiling (SAC) was used in 37.2\% (337/905) and balloon-assisted coiling (BAC) was used in 20.3\% (184/905).
The mean packing density was $32.3 \%$ (SD 18.21). The median time from first coil deployment to last coil detachment was $16.0 \mathrm{~min}$ (IQR 8.0, 31.0) and the median fluoroscopy time was $37.0 \mathrm{~min}$ (IQR 24.0, 55.0). In 80.7\% (729/903) of cases, no reaccess attempt was made due to catheter kick-out.

\section{Performance Outcomes}

Adequate occlusion at immediate post-procedure was achieved in $97.1 \%(879 / 905)$ of patients. Catheter kick-back (requiring re-access at least once or twice) occurred in $8.2 \%$ (74/903) of cases. The first framing coil was completely conformed to the lesion morphology in $83.3 \%$ (724/869) of cases. The immediate post-procedure RROC Class I or II was reported in $79.7 \%$ (717/900) of all aneurysm cases and at 1-year was 90\% (641/712). The overall 1-year retreatment rate was $7.1 \%(52 / 731)$ and the recanalization rate was $12.4 \%$. RROC I or II at 1 -year was achieved in $84.3 \%(161 / 191)$ of ruptured aneurysms and in $92.1 \%(480 / 521)$ of unruptured aneurysms. The retreatment rate for ruptured aneurysms $(N=288)$ at 1 -year was $16.5 \%$ $(33 / 200)$ and for unruptured aneurysms was $3.6 \%(19 / 531)$. The retreatment rate for wide-neck aneurysm (WNA, $N=554$ ) at 1 -year was $7.4 \%(34 / 458)$ compared to $6.4 \%(16 / 251)$ for nonWNA. Supplementary Table 1 shows the outcomes for RROC at 1 -year by immediate post-procedure RROC.

\section{Safety Outcomes}

Procedural device-related SAEs occurred in 2.9\% (26/905) of patients and one patient died within $24 \mathrm{~h}$ of the procedure 
TABLE 1 | Demographics and baseline characteristics.

\begin{tabular}{|c|c|c|c|}
\hline & $\begin{array}{l}\text { All patients } \\
(N=995)\end{array}$ & $\begin{array}{l}\text { Aneurysm } \\
\text { patients } \\
(N=905)\end{array}$ & $\begin{array}{c}\text { Non-aneurysm } \\
\text { patients } \\
(N=90)\end{array}$ \\
\hline Age, years [average (SD)] & $\begin{array}{l}59.6(13.00) \\
(N=995)\end{array}$ & $\begin{array}{l}59.8(12.64) \\
(N=905)\end{array}$ & $\begin{array}{l}57.9(16.18) \\
\quad(N=90)\end{array}$ \\
\hline Age $\geq 65$ years & $\begin{array}{c}38.3 \% \\
(381 / 995)\end{array}$ & $\begin{array}{c}38.0 \% \\
(344 / 905)\end{array}$ & $\begin{array}{l}41.1 \% \\
(37 / 90)\end{array}$ \\
\hline Female & $\begin{array}{c}72.1 \% \\
(717 / 995)\end{array}$ & $\begin{array}{c}74.7 \% \\
(676 / 905)\end{array}$ & $\begin{array}{l}45.6 \% \\
(41 / 90)\end{array}$ \\
\hline Hispanic or latino ethnicity & $\begin{array}{c}10.1 \% \\
(33 / 326)\end{array}$ & $\begin{array}{c}10.4 \% \\
(31 / 299)\end{array}$ & $\begin{array}{l}7.4 \% \\
(2 / 27)\end{array}$ \\
\hline \multicolumn{4}{|l|}{ Race } \\
\hline $\begin{array}{l}\text { American Indian or Alaska } \\
\text { Native }\end{array}$ & $\begin{array}{c}1.2 \% \\
(4 / 326)\end{array}$ & $\begin{array}{c}1.0 \% \\
(3 / 299)\end{array}$ & $\begin{array}{l}3.7 \% \\
(1 / 27)\end{array}$ \\
\hline Asian & $\begin{array}{c}1.8 \% \\
(6 / 326)\end{array}$ & $\begin{array}{l}2.0 \% \\
(6 / 299)\end{array}$ & $0.0 \%$ \\
\hline Black or African American & $\begin{array}{c}13.2 \% \\
(43 / 326)\end{array}$ & $\begin{array}{c}12.7 \% \\
(38 / 299)\end{array}$ & $\begin{array}{l}18.5 \% \\
(5 / 27)\end{array}$ \\
\hline White & $\begin{array}{c}78.5 \% \\
(256 / 326)\end{array}$ & $\begin{array}{c}78.6 \% \\
(235 / 299)\end{array}$ & $\begin{array}{l}77.8 \% \\
(21 / 27)\end{array}$ \\
\hline $\begin{array}{l}\text { Native Hawaiian or Other } \\
\text { Pacific Islander }\end{array}$ & $0.0 \%$ & $0.0 \%$ & $0.0 \%$ \\
\hline Other & $\begin{array}{c}5.2 \% \\
(17 / 326)\end{array}$ & $\begin{array}{c}5.7 \% \\
(17 / 299)\end{array}$ & $0.0 \%$ \\
\hline \multicolumn{4}{|l|}{ Medical history } \\
\hline $\begin{array}{l}\text { Previous hemorrhagic } \\
\text { stroke }\end{array}$ & $\begin{array}{c}8.8 \% \\
(88 / 995)\end{array}$ & $\begin{array}{c}9.6 \% \\
(87 / 905)\end{array}$ & $\begin{array}{l}1.1 \% \\
(1 / 90)\end{array}$ \\
\hline Previous ischemic stroke & $\begin{array}{c}6.1 \% \\
(61 / 995)\end{array}$ & $\begin{array}{c}6.3 \% \\
(57 / 905)\end{array}$ & $\begin{array}{l}4.4 \% \\
(4 / 90)\end{array}$ \\
\hline Hypertension & $\begin{array}{c}60.6 \% \\
(603 / 995)\end{array}$ & $\begin{array}{c}61.8 \% \\
(559 / 905)\end{array}$ & $\begin{array}{l}48.9 \% \\
(44 / 90)\end{array}$ \\
\hline $\begin{array}{l}\text { Family history of aneurysm } \\
\text { or malformation }\end{array}$ & $\begin{array}{c}17.1 \% \\
(170 / 995)\end{array}$ & $\begin{array}{c}18.3 \% \\
(166 / 905)\end{array}$ & $\begin{array}{l}4.4 \% \\
(4 / 90)\end{array}$ \\
\hline Diabetes & $\begin{array}{c}14.1 \% \\
(140 / 995)\end{array}$ & $\begin{array}{c}14.1 \% \\
(128 / 905)\end{array}$ & $13.3 \%(12 / 90)$ \\
\hline Smoking (current or former) & $\begin{array}{c}61.2 \% \\
(609 / 995)\end{array}$ & $\begin{array}{c}63.0 \% \\
(570 / 905)\end{array}$ & $\begin{array}{l}43.3 \% \\
(39 / 90)\end{array}$ \\
\hline Polycystic kidney disease & $\begin{array}{l}0.6 \% \\
(6 / 995)\end{array}$ & $\begin{array}{l}0.7 \% \\
(6 / 905)\end{array}$ & $0.0 \%$ \\
\hline
\end{tabular}

(described above in "All Patient Results"). The mortality rate at 1-year was 5.4\% (49/905); $11.8 \%$ for patients with ruptured aneurysms and $2.4 \%$ for patients with unruptured aneurysms. At 1-year, there were $84.4 \%(429 / 508)$ of patients with mRS $0-2 ; 65.3 \%$ for ruptured aneurysms and $92.2 \%$ in unruptured aneurysms. The procedural device-related SAE rate for patients treated for ruptured aneurysms was 3.1\% (9/288) and for unruptured aneurysms was $2.8 \%$ (17/617). In patients with WNA, the procedural device-related SAEs rate was $2.5 \%(\mathrm{n} / \mathrm{N})$ and this rate for non-WNA was $3.8 \%(12 / 318)$.

\section{Predictive Analysis}

Predictors of 1-year for RROC III or retreatment (incomplete occlusion) were rupture status (OR 3.61, 95\% CI 2.275.74, $P<0.0001$ ), balloon-assisted coiling (OR 1.72, 95\%
TABLE 2 | Lesion characteristics.

\begin{tabular}{|c|c|c|c|}
\hline & $\begin{array}{l}\text { All patients } \\
(N=995)\end{array}$ & $\begin{array}{l}\text { Aneurysm } \\
\text { patients } \\
(N=905)\end{array}$ & $\begin{array}{c}\text { Non-aneurysm } \\
\text { patients } \\
(N=90)\end{array}$ \\
\hline Fistula & - & - & $\begin{array}{l}45.6 \% \\
(41 / 90)\end{array}$ \\
\hline Arteriovenous malformation & - & - & $\begin{array}{l}12.2 \% \\
(11 / 90)\end{array}$ \\
\hline Vessel sacrifice or pseudoaneurysm & - & - & $\begin{array}{l}42.2 \% \\
(38 / 90)\end{array}$ \\
\hline \multicolumn{4}{|l|}{ Lesion location } \\
\hline Extradural ICA & $\begin{array}{c}3.7 \% \\
(37 / 995)\end{array}$ & $\begin{array}{c}2.2 \% \\
(20 / 905)\end{array}$ & $\begin{array}{l}18.9 \% \\
(17 / 90)\end{array}$ \\
\hline Intradural ICA & $\begin{array}{c}33.0 \% \\
(328 / 995)\end{array}$ & $\begin{array}{c}36.1 \% \\
(327 / 905)\end{array}$ & $\begin{array}{l}1.1 \% \\
(1 / 90)\end{array}$ \\
\hline ACA & $\begin{array}{c}26.7 \% \\
(266 / 995)\end{array}$ & $\begin{array}{c}29.3 \% \\
(265 / 905)\end{array}$ & $\begin{array}{l}1.1 \% \\
(1 / 90)\end{array}$ \\
\hline MCA & $\begin{array}{c}11.8 \% \\
(117 / 995)\end{array}$ & $\begin{array}{c}12.5 \% \\
(113 / 905)\end{array}$ & $\begin{array}{l}4.4 \% \\
(4 / 90)\end{array}$ \\
\hline Posterior circulation & $\begin{array}{c}18.9 \% \\
(188 / 995)\end{array}$ & $\begin{array}{c}19.8 \% \\
(179 / 905)\end{array}$ & $\begin{array}{l}10.0 \% \\
(9 / 90)\end{array}$ \\
\hline Venous circulation & $\begin{array}{c}2.4 \% \\
(24 / 995)\end{array}$ & $\begin{array}{c}0.1 \% \\
(1 / 905)\end{array}$ & $\begin{array}{l}25.6 \% \\
(23 / 90)\end{array}$ \\
\hline Other & $\begin{array}{c}3.5 \% \\
(35 / 995)\end{array}$ & $0.0 \%$ & $\begin{array}{l}38.9 \% \\
(35 / 90)\end{array}$ \\
\hline \multicolumn{4}{|l|}{ Lesion laterality } \\
\hline Left & $\begin{array}{c}35.6 \% \\
(354 / 995)\end{array}$ & $\begin{array}{c}33.9 \% \\
(307 / 905)\end{array}$ & $\begin{array}{l}52.2 \% \\
(47 / 90)\end{array}$ \\
\hline Right & $\begin{array}{c}35.6 \% \\
(354 / 995)\end{array}$ & $\begin{array}{c}34.8 \% \\
(315 / 905)\end{array}$ & $\begin{array}{l}43.3 \% \\
(39 / 90)\end{array}$ \\
\hline Midline & $\begin{array}{c}28.8 \% \\
(287 / 995)\end{array}$ & $\begin{array}{c}31.3 \% \\
(283 / 905)\end{array}$ & $\begin{array}{l}4.4 \% \\
(4 / 90)\end{array}$ \\
\hline \multicolumn{4}{|l|}{ Aneurysm characteristics } \\
\hline Aneurysm size, mm [average (SD)] & - & $\begin{array}{c}6.9 \\
(3.62)(N=905)\end{array}$ & - \\
\hline \multicolumn{4}{|l|}{ Aneurysm size group } \\
\hline Small $(\leq 4 \mathrm{~mm})$ & - & $\begin{array}{c}19.0 \% \\
(172 / 905)\end{array}$ & - \\
\hline Medium (>4-10 mm) & & $\begin{array}{c}66.3 \% \\
(600 / 905)\end{array}$ & \\
\hline Large (>10-25 mm) & - & $\begin{array}{c}14.5 \% \\
(131 / 905)\end{array}$ & - \\
\hline Giant (>25 mm) & - & $\begin{array}{c}0.2 \% \\
(2 / 905)\end{array}$ & - \\
\hline \multicolumn{4}{|l|}{ Aneurysm type } \\
\hline Saccular & - & $\begin{array}{c}86.3 \% \\
(778 / 901)\end{array}$ & - \\
\hline Dissecting & & $\begin{array}{c}2.6 \% \\
(23 / 901)\end{array}$ & \\
\hline Fusiform & & $\begin{array}{c}2.4 \% \\
(22 / 901)\end{array}$ & \\
\hline Other & & $\begin{array}{c}8.7 \% \\
(78 / 901)\end{array}$ & \\
\hline Wide neck & - & $\begin{array}{c}63.5 \% \\
(554 / 872)\end{array}$ & - \\
\hline Ruptured & - & $\begin{array}{c}31.8 \% \\
(288 / 905)\end{array}$ & - \\
\hline Hunt and Hess $\geq 3$ & - & $\begin{array}{c}43.5 \% \\
(123 / 283)\end{array}$ & - \\
\hline
\end{tabular}

\section{Aneurysm characteristics}

Aneurysm size group 
TABLE 3 | Procedural characteristics.

\begin{tabular}{|c|c|c|c|}
\hline & $\begin{array}{l}\text { All patients } \\
(N=995)\end{array}$ & $\begin{array}{l}\text { Aneurysm } \\
\text { patients } \\
(N=905)\end{array}$ & $\begin{array}{l}\text { Non-aneurysm } \\
\text { patients } \\
(N=90)\end{array}$ \\
\hline \multicolumn{4}{|l|}{ Adjunctive device use } \\
\hline Unassisted coiling & $\begin{array}{c}44.8 \% \\
(446 / 995)\end{array}$ & $\begin{array}{c}43.3 \% \\
(392 / 905)\end{array}$ & $\begin{array}{l}60.0 \% \\
(54 / 90)\end{array}$ \\
\hline Stent-assisted coiling & $\begin{array}{c}34.4 \% \\
(342 / 995)\end{array}$ & $\begin{array}{c}37.2 \% \\
(337 / 905)\end{array}$ & $\begin{array}{l}5.6 \% \\
(5 / 90)\end{array}$ \\
\hline Balloon-assisted coiling & $\begin{array}{c}18.7 \% \\
(186 / 995)\end{array}$ & $\begin{array}{c}20.3 \% \\
(184 / 905)\end{array}$ & $\begin{array}{l}2.2 \% \\
(2 / 90)\end{array}$ \\
\hline Flow diverter & $\begin{array}{c}1.6 \% \\
(16 / 995)\end{array}$ & $\begin{array}{c}1.8 \% \\
(16 / 905)\end{array}$ & $\begin{array}{l}0.0 \% \\
(0 / 90)\end{array}$ \\
\hline Liquid embolic & $\begin{array}{c}2.1 \% \\
(21 / 995)\end{array}$ & $\begin{array}{l}0.1 \% \\
(1 / 905)\end{array}$ & $\begin{array}{l}22.2 \% \\
(20 / 90)\end{array}$ \\
\hline Particulate/plug embolic & $\begin{array}{c}1.2 \% \\
(12 / 995)\end{array}$ & $\begin{array}{c}0.3 \% \\
(3 / 905)\end{array}$ & $\begin{array}{l}10.0 \% \\
(9 / 90)\end{array}$ \\
\hline $\begin{array}{l}\text { Number of coils implanted } \\
\text { [SMART/PC400/POD, } \\
\text { average (SD)] }\end{array}$ & $\begin{array}{l}5.4(5.03) \\
(N=994)\end{array}$ & $\begin{array}{l}5.0(3.88) \\
(N=904)\end{array}$ & $\begin{array}{c}9.3(10.62) \\
(N=90)\end{array}$ \\
\hline $\begin{array}{l}\text { Packing density, \% [average } \\
\text { (SD)] }\end{array}$ & - & $\begin{array}{l}32.3(18.21) \\
(N=819)\end{array}$ & - \\
\hline \multicolumn{4}{|l|}{ Procedural times } \\
\hline $\begin{array}{l}\text { Fluoroscopic time, min } \\
\text { [median (IQR)] }\end{array}$ & $\begin{array}{c}37.0(24.0,56.0) \\
\quad(N=992)\end{array}$ & $\begin{array}{c}37.0(24.0,55.0) \\
\quad(N=902)\end{array}$ & $\begin{array}{c}41.0(25.0,73.0) \\
(N=90)\end{array}$ \\
\hline $\begin{array}{l}\text { Time from first coil } \\
\text { deployment to last coil } \\
\text { detachment, min [median } \\
\text { (IQR)] }\end{array}$ & $\begin{array}{c}16.0(8.0,32.0) \\
(N=952)\end{array}$ & $\begin{array}{c}16.0(8.0,31.0) \\
\quad(N=868)\end{array}$ & $\begin{array}{c}20.5(3.0,53.0) \\
\quad(N=84)\end{array}$ \\
\hline
\end{tabular}

CI $1.04-2.87, P=0.0354$ ), aneurysm size (OR 2.23, 95\% CI 1.24-4.00, $P=0.0071$ ), and incomplete occlusion immediate post-procedure (OR 2.01, 95\% CI 1.19-3.37, $P=0.0086)$. Predictor of aneurysm retreatment at 1 year was rupture status (OR 5.32, 95\% CI 2.95-9.61, $P<0.0001)$.

\section{Non-aneurysm Cohort Outcomes}

Of the 90 patients treated for non-aneurysms; 45.6\% (41/90) were fistulas, $12.2 \%$ (11/90) were arteriovenous malformations, and $42.2 \%(38 / 90)$ were vessel sacrifice or pseudoaneurysm. In non-aneurysm treated patients, UAC was used in $60.0 \%$ (54/90), liquid embolics in $22.2 \%(20 / 90)$, and $10.0 \%(9 / 90)$ particulate/plug embolics. $79.2 \%(42 / 53)$ of all patients had the first coil conformed to the lesion. The median time from first coil to last coil deployment was $20.5 \mathrm{~min}$ (IQR $3.0,53.0$ ) and the median fluoroscopy time was $41.0 \mathrm{~min}$ (IQR 25.0, 73.0). Retreatment at 1-year was $1.9 \%(1 / 53)$ and the recanalization rate was $6.1 \%(3 / 49)$. At 1 -year, $90.9 \%$ $(20 / 22)$ of fistula treated patients, $54.5 \%(12 / 22)$ of patients treated for other lesions, and $40 \%(2 / 5)$ of patients treated for malformation had mRS 0 -2. There were no procedural devicerelated SAEs $(0.0 \%)$ and the mortality rate within $24 \mathrm{~h}$ of the procedure was $1.1 \%(1 / 90)$. The mortality rate at 1 -year was $12.2 \%(11 / 90)$.
TABLE 4 | Study outcomes.

\begin{tabular}{|c|c|c|c|}
\hline & $\begin{array}{l}\text { All patients } \\
(N=995)\end{array}$ & $\begin{array}{l}\text { Aneurysm } \\
\text { patients } \\
(N=905)\end{array}$ & $\begin{array}{c}\text { Non-aneurysm } \\
\text { patients } \\
(N=90)\end{array}$ \\
\hline \multicolumn{4}{|l|}{ Peri-procedural outcomes } \\
\hline $\begin{array}{l}\text { Procedural device-related } \\
\text { serious adverse events } \\
\text { (SAEs) }\end{array}$ & $\begin{array}{c}2.6 \% \\
(26 / 995)\end{array}$ & $\begin{array}{c}2.9 \% \\
(26 / 905)\end{array}$ & $0.0 \%$ \\
\hline $\begin{array}{l}\text { All SAEs (intraprocedural or } \\
\text { within } 24 \mathrm{~h} \text { ) }\end{array}$ & $\begin{array}{c}9.4 \% \\
(94 / 995)\end{array}$ & $\begin{array}{c}9.7 \% \\
(88 / 905)\end{array}$ & $\begin{array}{l}6.7 \% \\
(6 / 90)\end{array}$ \\
\hline $\begin{array}{l}\text { Mortality within } 24 \mathrm{~h} \text { of } \\
\text { procedure }\end{array}$ & $\begin{array}{l}0.2 \% * \\
(2 / 995)\end{array}$ & $\begin{array}{c}0.1 \% \\
(1 / 905)\end{array}$ & $\begin{array}{l}1.1 \% \\
(1 / 90)\end{array}$ \\
\hline $\begin{array}{l}\text { Adequate occlusion at } \\
\text { immediate post-procedure }\end{array}$ & & $\begin{array}{c}97.1 \% \\
(879 / 905)\end{array}$ & \\
\hline $\begin{array}{l}\text { Complete angiographic } \\
\text { occlusion } \\
\text { immediate post-procedure }\end{array}$ & - & - & $\begin{array}{l}85.2 \% \\
(23 / 27)\end{array}$ \\
\hline \multicolumn{4}{|l|}{$\begin{array}{l}\text { Re-access attempts with } \\
\text { guidewire due to catheter } \\
\text { kickout } \\
\text { (SMART/PC400/POD) }\end{array}$} \\
\hline 0 & $\begin{array}{c}81.7 \% \\
(811 / 993)\end{array}$ & $\begin{array}{c}80.7 \% \\
(729 / 903)\end{array}$ & $\begin{array}{l}91.1 \% \\
(82 / 90)\end{array}$ \\
\hline 1 & $\begin{array}{c}10.6 \% \\
(105 / 993)\end{array}$ & $\begin{array}{c}11.1 \% \\
(100 / 903)\end{array}$ & $\begin{array}{l}5.6 \% \\
(5 / 90)\end{array}$ \\
\hline $2+$ & $\begin{array}{c}7.8 \% \\
(77 / 993)\end{array}$ & $\begin{array}{c}8.2 \% \\
(74 / 903)\end{array}$ & $\begin{array}{l}3.3 \% \\
(3 / 90)\end{array}$ \\
\hline \multicolumn{4}{|l|}{ Outcomes at 1-year } \\
\hline Retreatment & $\begin{array}{c}6.8 \% \\
(53 / 784)\end{array}$ & $\begin{array}{c}7.1 \% \\
(52 / 731)\end{array}$ & $\begin{array}{l}1.9 \% \\
(1 / 53)\end{array}$ \\
\hline RROC I or II & - & $\begin{array}{c}90.0 \% \\
(641 / 712)\end{array}$ & - \\
\hline Recanalization & $\begin{array}{c}12.4 \% \\
(94 / 757)\end{array}$ & $\begin{array}{c}12.9 \% \\
(91 / 708)\end{array}$ & $\begin{array}{l}6.1 \% \\
(3 / 49)\end{array}$ \\
\hline mRS 0-2 & $\begin{array}{c}83.1 \% \\
(463 / 557)\end{array}$ & $\begin{array}{c}84.4 \% \\
(429 / 508)\end{array}$ & $\begin{array}{l}69.4 \% \\
(34 / 49)\end{array}$ \\
\hline All-cause mortality & $\begin{array}{c}6.0 \% \\
(60 / 995)\end{array}$ & $\begin{array}{c}5.4 \% \\
(49 / 905)\end{array}$ & $\begin{array}{l}12.2 \% \\
(11 / 90)\end{array}$ \\
\hline \multicolumn{4}{|l|}{ All serious adverse events } \\
\hline $\begin{array}{l}\text { After } 24 \mathrm{~h} \text { through } 365 \\
\text { days }^{\dagger}\end{array}$ & $\begin{array}{c}22.7 \% \\
(226 / 995)\end{array}$ & $\begin{array}{c}22.2 \% \\
(201 / 905)\end{array}$ & $\begin{array}{l}27.8 \% \\
(25 / 90)\end{array}$ \\
\hline
\end{tabular}

*Not procedure-related or device-related.

${ }^{\dagger}$ Defined as adverse events that started after the date of registry completion.

\section{DISCUSSION}

The results of the SMART Registry provide clinical evidence of the treatment with SMART, PC400, and POD in intracranial aneurysms and other neurovascular abnormalities. Patients in this registry had low retreatment rates through 1 year. The procedural device-related SAE rate was low. A high rate of patients achieved adequate occlusion post-procedure and at 1 -year, and our rates were consistent or better than rates reported in other SMART COIL series $(5-8,11)$. Mortality within $24 \mathrm{~h}$ of the procedure occurred in two patients due to progression of baseline SAH from a ruptured aneurysm. The SMART Registry results confirm the safety and durability of 
the SMART System for treatment of intracranial aneurysms and other neurovascular abnormalities.

The SMART Registry outcomes are comparable to contemporary studies of other coils. The TARGET Registry was a prospective, single-arm registry that compared outcomes for patients with saccular (ruptured and unruptured) intracranial aneurysms treated exclusively with TARGET-360 (Stryker Corp., Fremont, California) complex shape coils (designated for framing, filling, and finishing) vs. patients treated with both complex shape and helical coils (12). There were no significant differences between the groups for complete or near complete occlusion and in retreatment rates at 6 months, or for any other safety or efficacy outcomes. In the combined group analysis, median packing density was $28.8 \%$, 6 -month RROC I or II was $90.4 \%$, and recanalization was $15.2 \%$. In comparison to the SMART Registry, the mean packing density was 32.3\%; and at 1-year, RROC I or II was $90 \%$ and recanalization was 12.9\%. Despite the SMART Registry having longer follow-up than the TARGET Registry ( $12 \pm 6$ months), the SMART Registry occlusion rate was comparable and the recanalization rate was lower than in TARGET registry. In addition, TARGET excluded fusiform and dissecting aneurysms as well as patients with severe aneurysm ruptures (Hunt and Hess $>3$ ), while in the SMART Registry 20.5\% (58/283) of patients had severe aneurysm ruptures.

In the prospective randomized GREAT Trial, intracranial aneurysms ranging $4-12 \mathrm{~mm}$ diameter were treated with either second generation hydrogel coils or platinum coils (13). There were no significant differences in procedural outcomes between the two coil types. The angiographic follow-up at 18 months showed that the platinum coil arm had adequate occlusion (per core lab RROC I + II) in $73 \%$ of patients and retreatment rate of $6 \%$. The hydrocoil arm had higher adequate occlusion $(80 \%)$ and a lower retreatment rate (3.0\%) than the platinum coil arm. In comparison, the SMART Registry rate of adequate occlusion at 12 months was higher (90.0\%) than both treatment arms in GREAT and retreatment rates for the SMART Registry and GREAT were similar. In a large systematic review including 8,161 aneurysms treated with standard and modified (hydrocoils and coated) coils, the retreatment rate was $10.3 \%$ (95\% CI 9.5\%, 11.0\%) (14).

The SMART Registry provides evidence of safety of treatment in aneurysms and other neurovascular lesions. Mortality within $24 \mathrm{~h}$ of the procedure in the overall population was low $(0.2 \%)$ and none were procedural or device-related. In the TARGET real-world randomized trial that evaluated outcomes after coiling aneurysms, the periprocedural mortality rate was $0.7 \%$ (12). In the GREAT trial for aneurysms, the 14 -day mortality was $2.1 \%$ in the bare platinum coil arm (13). In the SMART Registry, there were no device complications associated with an $\mathrm{AE}$ and safety was demonstrated across multiple complex lesion types. In the non-aneurysm subgroup of the SMART Registry, which included fistulas, arteriovenous malformations, pseudoaneurysms, and vessel sacrifices, there were no procedural device-related SAEs, adequate occlusion immediate post-procedure was high (96.6\%), and retreatment at 1-year was low (1.9\%). These results are consistent with previous POD studies and also alternative treatments (15-17).
The SMART Registry included several complex lesion subgroups, such as a large number of patients treated for wideneck aneurysms (WNA), ruptured aneurysms, and patients with non-saccular aneurysms (e.g., fusiform and dissecting). The patients treated for WNA had low procedural device-related safety events (2.5\%) and low retreatment rates (7.4\%) through 1 -year. These outcomes are consistent with other reports of coiled WNA (18-23). Patients with ruptured aneurysms in our study achieved high occlusion rates at 1-year (RROC I + II 84.3\%) and as expected, these patients had higher rates of recanalization and retreatment compared to those patients treated for unruptured aneurysms. These findings are consistent with previous reports in real-world registries treating ruptured aneurysms with endovascular coiling (24-26). The SMART ruptured aneurysm subgroup achieved occlusion comparable to other studies evaluating ruptured aneurysms treated with bare metal coils (24). Another study of ruptured aneurysms treated with coiling reported a periprocedural safety complication rate as high as $12.7 \%$ compared to $3.1 \%$ in the SMART Ruptured population (27). Therefore, SMART Coil appears to maintain safety and performance across multiple complex lesion types. Further investigation into complex subgroups from this study will be presented in subsequent reports from the SMART Registry.

The unique design of the SMART System features progressive coil softness and polymer technology that are not present in other commercially available coils. The coil tip is a softened loop and is designed to help reduce kickback and vessel perforations. The progressive coil softness potentially can lead to less compartmentalization and better packing density (28). Rigid coils are typically effective for framing, while softer coils are more effective for filling and finishing. The SMART COIL design decreases compartmentalization with stable framing of the aneurysm wall and supports durable occlusion through 1 year. In the SMART Registry, compartmentalization rate was low (2.0\%) and demonstrated the ability to treat a variety of complex lesion types and treat distal lesion locations safely, such as the anterior, mid, and posterior communicating arteries. Previous studies have suggested a correlation between coil packing density and recanalization (29). Increased packing density promotes accelerated healing and decrease recurrence and retreatment (30). In other neurovascular embolization coil studies, packing density has been reported between 27.4 and $37.9 \%(7,31-34)$. The mean packing density was $32.3 \%$ in the SMART Registry and this may have contributed to low rates of recanalization.

In this study population, we found that predictors at 1 year for retreatment was ruptured status. RROC III immediate post-procedure, aneurysm size, balloon-assisted coiling, and ruptured aneurysm were predictors for RROC III or retreatment (incomplete occlusion) at 1-year. The predictors for occlusion in the TARGET Registry were aneurysm location, size, and immediate occlusion grade (12).

The SMART Registry is one of the largest real-world prospective embolization coil studies completed to date and included a large sample size of complex lesions. The study included ruptured and unruptured aneurysms ranging from small to giant sizes, wide-neck and complex shaped 
aneurysms; and other malformations, including arterial fistula, malformations, and vessel embolization for treatment of tumors or hemorrhages. Heterogenicity of the patient and lesion population allows future insight into multiple complex subgroups and provides good reflection of current treatment techniques and long-term outcomes using contemporary coils. A key limitation of the study is the lack of a comparator arm. Another limitation was that this study did not have a centralized angiographic core lab performing independent occlusion assessments.

\section{CONCLUSION}

This study provides clinical evidence of the SMART System in real-world clinical settings. The findings demonstrated that the SMART System is safe and efficacious and substantiate the durability of the coils at 1 -year. Additional analysis of subgroups will be beneficial.

\section{DATA AVAILABILITY STATEMENT}

The original contributions presented in the study are included in the article/Supplementary Material, further inquiries can be directed to the corresponding author/s.

\section{ETHICS STATEMENT}

The studies involving human participants were reviewed and approved by all investigational study sites received approval and

\section{REFERENCES}

1. Keedy A. An overview of intracranial aneurysms. McGill J Med. (2006) 9:141-6. doi: 10.26443/mjm.v9i2.672

2. Thompson BG, Brown RD, Amin-Hanjani S, Broderick JP, Cockroft KM, Connolly ES, et al. Guidelines for the management of patients with unruptured intracranial aneurysms: a guideline for healthcare professionals from the American Heart Association/American Stroke Association. Stroke. (2015) 46:2368-400. doi: 10.1161/STR.0000000000000070

3. Campi A, Ramzi N, Molyneux AJ, Summers PE, Kerr RSC, Sneade M, et al. Retreatment of ruptured cerebral aneurysms in patients randomized by coiling or clipping in the international subarachnoid aneurysm trial (ISAT). Stroke. (2007) 38:1538-44. doi: 10.1161/STROKEAHA.106.466987

4. Daniel B, Henrik S, Ioannis T, Veit R, Marios-Nikos P. SMART coils for intracranial aneurysm repair - a single center experience. BMC Neurol. (2020) 20:38. doi: 10.1186/s12883-020-1623-9

5. Spiotta, Fargen K, Lena J, Chaudry I, Turner R, Turk A, et al. Progressively soft coils for treatment of intracerebral aneurysms-an initial experience with the smart coil at two centers. J Neurointerv Surg. (2017) 9:A66-7. doi: 10.1136/neurintsurg-2017-SNIS.101

6. Stapleton CJ, Torok CM, Patel AB. Early experience with the Penumbra SMART coil in the endovascular treatment of intracranial aneurysms: safety and efficacy. Interv Neuroradiol. (2016) 22:654-8. doi: 10.1177/1591019916663479

7. Ilyas A, Buell TJ, Chen CJ, Ding D, Raper DMS, Taylor DG, et al. SMART coils for intracranial aneurysm embolization: initial outcomes. Clin Neurol Neurosurg. (2018) 164:87-91. doi: 10.1016/j.clineuro.2017.11.020

8. Sokolowski JD, Ilyas A, Buell TJ, Taylor DG, Chen C-J, Ding D, et al. SMART coils for intracranial aneurysm embolization: follow-up outcomes. J Clin Neurosci. (2018) 59:93-7. doi: 10.1016/j.jocn.2018.10.132 oversite from their ethics committee. The patients/participants provided their written informed consent to participate in this study.

\section{AUTHOR CONTRIBUTIONS}

All authors were responsible for conducting research, data collection, analysis, interpretation, review of the article, final draft approval, and primary investigators at their institutions and were responsible for protocol execution within local and international regulatory requirements. AS was the Principal Investigator and lead author.

\section{ACKNOWLEDGMENTS}

We would like to thank the SMART Registry Investigators (see Supplementary Table 2 for full list of Investigators) and Study Coordinators for all of their contribution to the study. The authors would like to acknowledge Sam Watcha, MS, Statistician and Susan M. Bezenek, RN BA MPH, Senior Medical Writer for editorial support. They both received salary support from Penumbra Inc. for this work.

\section{SUPPLEMENTARY MATERIAL}

The Supplementary Material for this article can be found online at: https://www.frontiersin.org/articles/10.3389/fneur. 2021.637551/full\#supplementary-material
9. Roy D, Milot G, Raymond J. Endovascular treatment of unruptured aneurysms. Stroke. (2001) 32:1998-2004. doi: 10.1161/hs0901.095600

10. Hunt WE, Hess RM. Surgical risk as related to time of intervention in the repair of intracranial aneurysms. J Neurosurg. (1968) 28:14-20. doi: 10.3171/jns.1968.28.1.0014

11. McAvoy MB, Cappuzzo JM, Stapleton CJ, Koch MJ, Raymond SB, Torok $\mathrm{CM}$, et al. Long-term follow-up results of the SMART coil in the endovascular treatment of intracranial aneurysms. Interv Neuroradiol. (2020). doi: 10.1177/1591019920956890. [Epub ahead of print].

12. Zaidat OO, Castonguay AC, Rai AT, Badruddin A, Mack WJ, Alshekhlee AK, et al. TARGET $\AA$ intracranial aneurysm coiling prospective multicenter registry: final analysis of peri-procedural and long-term safety and efficacy results. Front Neurol. (2019) 10:737. doi: 10.3389/fneur.2019. 00737

13. Taschner CA, Chapot R, Costalat V, Machi P, Courthéoux P, Barreau X, et al. GREAT - a randomized controlled trial comparing HydroSoft/HydroFrame and bare platinum coils for endovascular aneurysm treatment: procedural safety and core-lab-assessedangiographic results. Neuroradiology. (2016) 58:777-86. doi: 10.1007/s00234-016-1693-y

14. Ferns SP, Sprengers MES, Van Rooij WJ, Rinkel GJE, Van Rijn JC, Bipat $S$, et al. Coiling of intracranial aneurysms: a systematic review on initial occlusion and reopening and retreatment rates. Stroke. (2009) 40:e523-9. doi: 10.1161/STROKEAHA.109.553099

15. Baharvahdat H, Blanc R, Fahed R, Smajda S, Ciccio G, Desilles JP, et al. Endovascular treatment for low-grade (Spetzler-Martin I-II) brain arteriovenous malformations. AJNR Am J Neuroradiol. (2019) 40:668-72. doi: 10.3174/ajnr.A5988

16. Baharvahdat H, Ooi YC, Kim WJ, Mowla A, Coon AL, Colby GP. Updates in the management of cranial dural arteriovenous fistula. Stroke Vasc Neurol. (2020) 5:50-8. doi: 10.1136/svn-2019-000269 
17. Jambon E, Petitpierre F, Brizzi V, Dubuisson V, Le Bras Y, Grenier N, et al. Proximal occlusion of medium-sized vessels with the penumbra occlusion device: a study of safety and efficacy. Cardiovasc Interv Radiol. (2017) 40:2105. doi: 10.1007/s00270-016-1480-5

18. Cui YF, Xu H, Zu MH, Gu YM, Zhang QQ, Wei N, et al. Clinical application of Solitaire $\mathrm{AB}$ stent in assisting coiling embolization for intracranial widenecked aneurysms. J Interv Radiol. (2013) 22:617-20. Available online at: https://www.europeanreview.org/article/8741 (accessed March 30, 2021).

19. Durst CR, Starke RM, Gaughen JR, Geraghty S, Kreitel KD, Medel R, et al. Single-center experience with a dual microcatheter technique for the endovascular treatment of wide-necked aneurysms. J Neurosurg. (2014) 121:1093-101. doi: 10.3171/2014.7.JNS132237

20. Gory B, Klisch J, Bonafé A, Mounayer C, Beaujeux R, Moret J, et al. Solitaire $\mathrm{AB}$ stent-assisted coiling of wide-necked intracranial aneurysms: mid-term results from the SOLARE study. Neurosurgery. (2014) 75:215-9. doi: 10.1227/NEU.0000000000000415

21. Gu DQ, Zhang X, Luo B, Long XA, Duan CZ. The effect of neuroform stentassisted coil embolization of wide-necked intracranial aneurysms and clinical factors on progressive aneurysm occlusion on angiographic follow-up. J Clin Neurosci. (2013) 20:244-7. doi: 10.1016/j.jocn.2012.01.053

22. Liu P, Lv X, Li Y, Lv M. Stent-assisted coiling of ruptured wide-necked intracranial aneurysms: a single-center experience of 218 consecutive patients. Neurol India. (2016) 64:S70-7. doi: 10.4103/0028-3886.178045

23. Song JH, Chang IB, Ahn JH, Kim JH, Oh JK, Cho BM. Angiographic results of wide-necked intracranial aneurysms treated with coil embolization: a single center experience. J Korean Neurosurg Soc. (2015) 57:250-7. doi: $10.3340 / \mathrm{jkns} .2015 .57 .4 .250$

24. Pierot L, Cognard C, Ricolfi F, Anxionnat R. Immediate anatomic results after the endovascular treatment of ruptured intracranial aneurysms: analysis in the CLARITY series. Am J Neuroradiol. (2010) 31:907-11. doi: 10.3174/ajnr.A1954

25. Cognard C, Pierot L, Ricolfi F, Anxionnat R. Long-term anatomical results after endovascular treatment of ruptured intracranial aneurysms with GDC and matrix coils: analysis of the clarity series. J NeuroInterv Surg. (2011) 3:A8. doi: 10.1136/neurintsurg-2011-010097.18

26. Dinç H, Halil Öztürk M, Sari A, Çakir E, Gazioglu G, Kuzeyli K. Coil embolization in 481 ruptured intracranial aneurysms: angiographic and clinical results. Diagn Interv Radiol. (2013) 19:165-72. doi: 10.4261/1305-3825.DIR.6197-12.1

27. Liu Y, Wang J, Lin L, Sang C, Lin Z, Pan Y, et al. Clinical study on complications of intracranial ruptured aneurysm embolization by stentassisted coil. Med Sci Monit. (2018) 24:8115-24. doi: 10.12659/MSM.911773

28. Zhao R, Liu J, McComas S, Guo J, Girdhar G. In-vitro pliability assessment of embolization coils for intracranial aneurysm treatment. J Neurol Sci. (2019) 406:116432. doi: 10.1016/j.jns.2019.116432
29. Slob MJ, Sluzewski M, van Rooij WJ. The relation between packing and reopening in coiled intracranial aneurysms: a prospective study. Neuroradiology. (2005) 47:942-5. doi: 10.1007/s00234-0051446-9

30. Brinjikji W, White PM, Nahser H, Wardlaw J, Sellar R, Cloft HJ, et al. HydroCoils reduce recurrence rates in recently ruptured medium-sized intracranial aneurysms: a subgroup analysis of the HELPS trial. Am J Neuroradiol. (2015) 36:1136-41. doi: 10.3174/ajnr.A4266

31. Griessenauer CJ, Adeeb N, Foreman PM, Gupta R, Patel AS, Moore J, et al. Impact of coil packing density and coiling technique on occlusion rates for aneurysms treated with stent-assisted coil embolization. World Neurosurg. (2016) 94:157-64. doi: 10.1016/j.wneu.2016.06.127

32. Tosello RT, Batista UC, Pereira BJA, Piske RL. Packing density necessary to reach a high complete occlusion rate in circumferential unruptured intracranial aneurysms treated with stent-Assisted coil embolization. Am J Neuroradiol. (2017) 38:1973-7. doi: 10.3174/ajnr.A5303

33. Zhang K, Wang ZL, Gao BL, Xue JY, Li TX, Zhao TY, et al. Use of a first large-sized coil versus conventional coils for embolization of cerebral aneurysms: effects on packing density, coil length, and durable occlusion. World Neurosurg. (2019) 127:e685-91. doi: 10.1016/j.wneu.2019. 03.242

34. Taschner C, Chapot R, Costalat V, Courthéoux P, Barreau X, Berge J, et al. GREAT - a randomized aneurysm trial. Design of a randomized controlled multicenter study comparing HydroSoft/HydroFrame and bare platinum coils for endovascular aneurysm treatment. Neuroradiology. (2015) 57:599-604. doi: $10.1007 / \mathrm{s} 00234-015-1501-0$

Conflict of Interest: This study was funded by Penumbra Inc. (Alameda USA). Both the sponsor and authors were involved in the design and conduct of the study; collection, management, analysis, and interpretation of the data; preparation, review, and approval of the manuscript; and decision to submit the manuscript for publication.

The reviewer AC declared a past co-authorship with one of the authors $\mathrm{OZ}$ to the handling editor.

Copyright (c) 2021 Spiotta, Park, Bellon, Bohnstedt, Yoo, Schirmer, DeLeacy, Fiorella, Woodward, Hawk, Nanda, Zaidat, Sunenshine, Liu, Kabbani, Snyder, Sivapatham, Dumont, Reeves, Starke and the SMART Registry Investigators. This is an open access article distributed under the terms of the Creative Commons Attribution License (CC BY). The use, distribution or reproduction in other forums is permitted, provided the original author(s) and the copyright owner(s) are credited and that the original publication in this journal is cited, in accordance with accepted academic practice. No use, distribution or reproduction is permitted which does not comply with these terms. 